Cahiers de philosophie de l'université de

\title{
Gustave Chpet et la tradition de la pensée religieuse en Russie
}

\section{Maryse Dennes}

\section{(2) OpenEdition}

1 Journals

Édition électronique

URL : https://journals.openedition.org/cpuc/977

DOI : 10.4000/cpuc.977

ISSN : 2677-6529

Éditeur

Presses universitaires de Caen

\section{Édition imprimée}

Date de publication : 31 octobre 2011

Pagination : 93-109

ISBN : 978-2-84133-385-1

ISSN : $1282-6545$

\section{Référence électronique}

Maryse Dennes, «Gustave Chpet et la tradition de la pensée religieuse en Russie », Cahiers de philosophie de l'université de Caen [En ligne], 48 | 2011, mis en ligne le 01 septembre 2020, consulté le 05 février 2023. URL : http://journals.openedition.org/cpuc/977 ; DOI : https://doi.org/10.4000/cpuc. 977

\section{(c) (†) (8)}

Creative Commons - Attribution - Pas d'Utilisation Commerciale 4.0 International - CC BY-NC 4.0 https://creativecommons.org/licenses/by-nc/4.0/ 


\section{Gustave Chpet et la tradition de la pensée religieuse en Russie ${ }^{1}$}

\section{$\mathrm{L}$}

'ÉTUDE DE L'INFLUENCE de Vladimir Soloviev et de la pensée religieuse russe sur les travaux de Gustave Chpet peut sembler un véritable défi compte tenu de la réserve, pour ne pas dire de la distance et parfois même de l'opposition de Chpet par rapport aux questions religieuses. Il nous semble néanmoins intéressant d'essayer de répondre à un tel défi et de montrer comment la pensée de Vladimir Soloviev a joué un rôle particulier dans la pensée de Chpet en lui donnant certaines caractéristiques et en participant ainsi à ce que l'on peut considérer comme l'apport de Chpet au développement de la philosophie classique. Le fait que Chpet, reconnu comme représentant de la philosophie académique et non religieuse en Russie, ait pu utiliser certains éléments de la tradition religieuse russe est par lui-même remarquable et nous conduit à nous interroger de nouveau sur ce qu'il énonçait, en 1922, au début de son Aperçu du développement de l'histoire de la philosophie russe:

La philosophie acquiert un caractère national non pas dans les réponses qu'elle donne - une réponse de nature scientifique est la même, en effet, pour tous les peuples et toutes les langues - mais dans la façon de poser les questions, dans leur choix, dans leurs modifications. L'intérêt pour un problème ou un autre, la façon de s'y rapporter ainsi que de se rapporter à l'un ou l'autre de ses aspects comportent un caractère local, ethnique, temporel, en aucune manière la forme et le contenu idéals des problèmes. C'est seulement en ce sens que l'on peut parler d'une science nationale, sinon, la résolution des questions scientifiques - qu'elles soient de nature philosophique, mathématique ou cristallographique - si elle se fait en tenant compte des goûts nationaux, des penchants et des humeurs, alors elle ne garde rien en elle de scientifique ${ }^{2}$.

1. Ce texte reprend des éléments déjà présentés dans Maryse Dennes, «Vladimir Solov’ev and the Legacy of Russian Religious Thought in the Works of Gustav Shpet», in Gustav Shpet's Contribution to Philosophy and Cultural Theory, G. Tihanov (dir.), West Lafayette, Perdue University Press, 2010, p. 115-124.

2. G. Špet, Očerk razvitija russkoj filosofii [Aperçu du développement de l'histoire de la philosophie russe], in Sočinenija [Euvres], Moskva, Pravda, 1989, p. 11-344 (p. 12). 
Pourtant, nous allons montrer qu'en fait, Chpet n'hésite pas à emprunter certains éléments, devenus classiques, de la pensée religieuse russe pour alimenter sa réflexion. Cela signifie-t-il, conformément à ce que nous venons de citer de lui, qu'il n'est pas arrivé à atteindre le caractère réellement universel de la réponse à certaines questions ? Cela reste encore à débattre, mais simultanément nous pouvons dire que, dans ses écrits, conformément toujours à ce que nous venons de citer de lui, ces éléments de la pensée religieuse, qu'il a, pour certains, empruntés à Vladimir Soloviev, sont ainsi entrés dans l'héritage de la philosophie académique et ont acquis, grâce à cela, une portée quasi universelle.

L'un des problèmes importants de l'œuvre de Chpet est celui du «Moi ${ }^{3}$. Or, c'est en essayant de définir la spécificité du «Moi» que Chpet a eu recours à un concept devenu classique de la pensée russe religieuse, le concept de «sobornost' ${ }^{4}$. Si nous voulons rester fidèles aux critères que Chpet a lui-même avancés pour définir ce qui est réellement scientifique et universel, nous pourrions dire que, dans ce domaine-là, le philosophe russe, utilisant pour ce faire un concept emprunté à la pensée religieuse, un concept qui ne s'était pas encore totalement libéré de son caractère national, n'aurait donc pas accédé à une réponse définitive relative à la question du «Moi».

Néanmoins, l'usage du concept de «sobornost'» joue un rôle indiscutable dans l'élaboration de la réponse de Chpet. Il lui permet tout d'abord de se distinguer de tous les représentants de la philosophie classique occidentale, et, en particulier, de tous ceux qui dans la ligne de Kant, de Fichte et dans le contexte du protestantisme avaient mis en avant le «Moi» en tant que principe premier de la philosophie et s'étaient ainsi faits les tenants du subjectivisme.

Chpet relève les traces d'un tel subjectivisme. En 1916, dans La conscience et son propriétaire ${ }^{5}$, il va même jusqu'à les repérer chez les philosophes qui prétendaient pourtant eux-mêmes critiquer un tel subjectivisme, chez Natorp, Husserl, et même chez le philosophe russe N. Losski ${ }^{6}$. Nous avons affaire ici à un exemple significatif: en se tournant vers les penseurs russes

3. Voir à ce sujet, V. Moltchanov, «Le problème du "Moi" chez Gustave Chpet: l'expérience et l'expérimentation", Slavica occitania [Toulouse], n 26, 2008, Gustave Chpet et son héritage. Aux sources russes du structuralisme et de la sémiotique, M. Dennes (dir.), p. 213-224.

4. Ce concept est parfois traduit par «conciliarité». Nous préférons, ici, lui laisser sa forme russe translittérée.

5. G. Špet, Soznanie i ego sobstvennik [La conscience et son propriétaire], in Filosofskie etjudy [Études philosophiques], Moskva, Progress, 1994, p. 20-116; in Philosophia Natalis. Izbrannye psihologo-pedagogičeskie trudy [Euvres choisies de psychologie et pédagogie], Moskva, Rosspen, 2006, p. 264-310.

6. Ibid., p. 59 sq.; p. 282 sq. 
religieux et en leur empruntant un concept traditionnel et spécifique de leur orientation de pensée, Chpet trouve un élément lui permettant d'ouvrir de nouvelles perspectives dans la recherche d'une réponse à une question bien déterminée se rapportant à l'essence du «Moi », et, par voie de conséquence, à ce que l'on peut entendre par «conscience».

En dehors de Vladimir Soloviev, dont il est question dans La conscience et son propriétaire, les penseurs russes auxquels se réfère Chpet pour parler de cette question sont Pavel Florensky, Eugène et Serge Troubetzkoy. Nous nous appuierons, en particulier, sur ce qu'il en dit dans Le phénomène et le sens ${ }^{7}$ (pour Pavel Florensky) et dans La conscience et son propriétaire (pour Eugène et Serge Troubetzkoy).

En général, les références à Soloviev sont rares dans les écrits de Chpet. Comme nous venons de le mentionner, nous les trouvons surtout dans La conscience et son propriétaire, et, au premier abord, ces références ne semblent pas concerner le problème que nous avons mentionné, celui du «Moi». Elles se présentent plutôt comme des formes de remarques, faites en passant, pour justifier la démarche adoptée. Chpet renvoie tout d'abord à Soloviev lorsque, traitant du problème de la conscience, il recherche une justification de la forme et du contenu du problème qu'il a choisi de traiter.

Citons un extrait, en relevant bien l'importance que Chpet attribue à la différence entre question posée et réponse proposée, et à la méthode générale de son questionnement philosophique. Chpet introduit ce passage en parlant d'un avertissement fait par Soloviev, et il renvoie à un extrait de son article, «Premier principe de la philosophie théorique», publié en 1897 dans Questions de philosophie et de psychologie ${ }^{8}$ :

Je rappellerai surtout un avertissement de V. Soloviev: «Il ne s'agit pas seulement du fait que toute réponse doit être vérifiée par une pensée rigoureuse, mais du fait que cela est exigé aussi pour toute question. Dans la vie courante, on peut demander sans trop réfléchir: à qui appartient le manteau? Ou les chaussures? Mais de quel droit pouvons-nous demander, en philosophie: à qui appartient la conscience? - tout en supposant par là même la présence de différents Qui, auxquels il faut attribuer une conscience selon un mode privé ou collectif de propriété? La question elle-même correspond seulement à une expression philosophiquement inacceptable, celle de la certitude

7. G. Špet, Javlenie i smysl, fenomenologija kak osnovnaja nauka i ee problemy [Le phénomène et le sens, la phénoménologie comme science fondamentale et ses problèmes], Moskva, Rosspen, Hermes, 1914; in Mysl' i slovo, Izbrannye trudy [La pensée et le mot. Euvres choisies], Moskva, Rosspen, 2005, p. 35-180.

8. G. Špet, «Pervoe načalo teoretičeskoj filosofii » [«Premier principe de la philosophie théorique »], Voprosy filosofii i psihologii [Questions de philosophie et de psychologie], nº 40, 1897 , p. 910-911. 
dogmatique que des individus séparés ont un être propre et indépendant. Mais c'est précisément cette certitude qui a besoin d'être vérifiée et justifiée par les conclusions logiques indiscutables que l'on peut tirer de données évidentes [...]. Dans l'état actuel des choses, à la question : à qui appartient la conscience? Ou bien à qui appartiennent certains faits psychiques déterminés, constituant le point de départ d'une réflexion philosophique? On peut et on doit répondre que l'on ne sait pas ${ }^{9}$.

Il est intéressant de remarquer ici qu'immédiatement après cette citation de Soloviev, Chpet réaffirme que le «Moi» est précisément un "problème» et, en aucun cas, "un fondement ou un principe ${ }^{10}$.

Cela signifie que, se tournant vers Soloviev, Chpet cherche dans la pensée russe religieuse, une confirmation du fait que, posant une question, il ne propose pas pour autant une réponse définitive. Parallèlement à cela, le recours à Soloviev lui permet d'insister sur la signification de la question en tant que telle: dans la problématique du «Moi» et dans celle de la conscience, les réponses, selon lui, "pseudo-scientifiques», de la plupart des philosophes occidentaux, ne correspondent pas à la réalité. C'est pour cette raison qu'il ne faut pas considérer ces réponses comme scientifiques et à valeur universelle, mais qu'il faut, nous dit-il, les soumettre à la critique, de telle façon que nous puissions poser une nouvelle question, d'un point de vue autre, ce dernier pouvant être lui-même issu de l'usage que l'on peut faire de la pensée russe.

Nous comprenons que dans un tel contexte, les paroles précédemment citées de Soloviev se trouvent justifiées : pour Chpet, le subjectivisme n'est pas la démarche à retenir pour traiter du problème du «Moi». Chpet considère qu'il vaut mieux se tourner vers une expérience de l'être plus profonde et originelle. Il pense qu'il faut chercher de nouveaux principes, et que cette quête doit être conduite à partir d'une expérience nationale

9. G. Špet, Soznanie i ego sobstvennik [La conscience et son propriétaire], p. 76-77; p. 291 : «Напомню только предостережение В. Соловьева: “Дело в том, что не только всякий ответ должен быть проверен отчетливой мыслью, но то же требуется и от всякого вопроса. В житейском обиходе, можно не задумываясь спрашивать: чей кафтан? Или чьи калоши? Но по какому праву можем мы спрашивать в философии: чье сознание? - тем самым предлагая подлинное присутствие разных Кто, которым нужно отдать сознание в частную или общинную собственность? Самый вопрос есть лишь философски недопустимое выражение догматической уверенности в безотносительном и самотождественном бытии единичных существ. Но именно эта-то уверенность и требует проверки и оправдания через непреложные логические выводы из самоочевидных данных... - При настоящем положении дел, на вопрос, чье это сознание, или кому принадлежат данные психические факты, составляющие исходную точку философского рассуждения, можно и должно отвечать: неизвестно" ».

10. Ibid., p. $77 ;$ p. 291. 
concrète et spécifique. C'est pour cette raison que Chpet se réfère ici à Soloviev: non seulement pour justifier la question elle-même et la façon de la poser, mais aussi pour ouvrir de nouvelles perspectives dans la réponse à apporter.

Le deuxième recours à Soloviev correspond encore, chez Chpet, à une recherche de justification. Voici ce qu'il écrit, toujours dans La conscience et son propriétaire:

V. Soloviev avait profondément raison quand il confrontait cette question [il s'agit ici de la question: à qui appartient la conscience? - M. D.] aux questions: à qui appartient le manteau, à qui appartiennent les chaussures ${ }^{11}$ ?

Cependant, dans ce passage, Chpet va un peu plus loin et poursuit au sujet de la conscience:

Si par conscience et unité de la conscience nous entendons un objet idéal, c'est-à-dire si nous l'envisageons comme une essence, alors la question «à qui elle appartient?» est dépourvue de sens ${ }^{12}$.

Il est évident que, pour Chpet, la conscience n'est pas quelque chose qui appartient à un individu particulier, à un «Moi» personnel. Si la conscience est définie comme «edinstvo» («unité»), alors elle correspond à un objet idéal, et pour cette raison, elle concerne, en qualité d'unité, une pluralité de sujets. Elle est, nous dit Chpet «kollektiv i sobranie» («un collectif et une réunion").

Dans la suite de son exposé, Chpet ne mentionne plus Soloviev. Cela signifie-t-il qu'il le met de côté ou l'oublie? L'ensemble de la démarche nous conduit à penser que non. Dans la note relative à la première citation, Chpet met l'accent sur le caractère inachevé de l'œuvre citée de Soloviev, et il exprime son désir de tirer toutes les conséquences possibles des prémisses et des éléments de la pensée solovievienne, avancés dans ce travail:

Chez Soloviev, la question est envisagée à travers le lien étroit qu'elle entretient avec la question sur la réalité de l'unité de la conscience. C'est délibérément qu'ici je ne l'aborde pas, ne préférant indiquer qu'une partie de mes notes sur la question de l'unité de la conscience. Mais je veux aussi revenir à ce thème et reprendre en considération les articles de Vl. Soloviev et les répliques de L. M. Lopatine. Alors qu'il renouvelait récemment la discussion sur cette question, le Prince E. N. Troubetzkoy n’a pas, à mon avis, pris en compte

11. Ibid., p. 108; p. 306 : «В. Соловьев был глубоко прав, сопоставляя этот вопрос [речь идет здесь о вопросе чье сознание - M. D.] с вопросами: чей кафтан, чьи калоши?».

12. Ibid.: «Если мы под сознанием и его единством понимаем идеальный предмет, т.е. рассматриваем его в его сущности, то лишено смысла спрашивать, чье оно ». 
toutes les conclusions que l'on peut tirer des présupposés contenus dans les travaux de Vl. Soloviev qui n'ont pas été terminés ${ }^{13}$.

Cette note révèle bien l'intention de Chpet. Il s'agit, pour lui, d'examiner les prémisses de la pensée de Vladimir Soloviev, qui, jusqu’à son époque, n'auraient, selon lui, jamais été mises en lumière. Nous comprenons que, dans un tel contexte, pour Chpet, le fait de s'adresser à d'autres penseurs russes, héritiers de la pensée de Soloviev, puisse aider à éclaircir ce qui est resté caché dans le texte de Soloviev, et même à comprendre pourquoi cela est resté caché. Ainsi, en examinant les références de Chpet aux autres penseurs russes religieux du début du $\mathrm{XX}^{\mathrm{e}}$ siècle, nous ne penserons pas simplement à leur influence proprement dite, mais aussi au rôle qu'ils ont pu jouer dans l'éclaircissement de certains aspects de la pensée de Soloviev.

Dans cette optique, il est intéressant de se tourner vers le texte fondamental de l'œuvre de Chpet, écrit en 1914, soit deux ans avant La conscience et son propriétaire. Nous voulons parler du texte déjà cité du Phénomène et le sens.

C'est précisément à la fin du dernier chapitre (le chapitre 7, si important, puisque c'est dans ce chapitre que se trouvent donnés, pour la première fois, les éléments de la structure du mot et de l'expression ${ }^{14}$ ), que Chpet se réfère à l'un des plus grands représentants de la pensée russe religieuse de son époque, Pavel Florensky, qu'il tient dans une grande estime. Le renvoi à Florensky se fait ici sous la forme d'une diversion, parallèlement au développement du thème principal du chapitre et en retrait des réflexions philosophiques proprement dites. Il s'agit là encore d'une note de bas de page, mais d'une longue note qui mérite d'être étudiée séparément et analysée parallèlement au contenu du chapitre et du livre dans son ensemble. Le contenu du texte de la note est apparemment hermétique. La présentation que nous voulons en faire a pour but de montrer comment Chpet parvient à établir un lien

13. G. Špet, Soznanie i ego sobstvennik [La conscience et son propriétaire], p. 77, n. 18; p. 291, n. 18: «У Соловьёва вопрос рассматривается в тесной связи с вопросом о реальности единства сознания. Я намеренно пока этого вопроса не касаюсь, помещая здесь только часть своих заметок по вопросу о единстве сознания. Но я хочу ещё вернуться к этой теме и подробно рассмотреть статьи Вл. Соловьёва и реплики на них Л.М. Лопатина. Кн. Е.Н. Трубецкой, возобновивший недавно спор по этим вопросам, на мой взгляд, не предусмотрел всех выводов, которые ещё можно сделать из предпосылок недоконченной работы Вл. Соловьёва».

14. Sur l'importance de cette formulation qui a été à la base de toutes les réflexions ultérieures de Gustave Chpet, et que l'on peut considérer comme un élément fondateur, bien que longtemps ignoré, du structuralisme et de la sémiotique, voir : M. Dennes, «L'influence de Husserl en Russie au début du XX ${ }^{\mathrm{e}}$ siècle et son impact sur les émigrés russes de Prague», Cahiers de l'ILSL [Lausanne] (en collaboration avec les Cahiers Roman Jakobson), 9, 1997, Jakobson entre l'Est et l'Ouest, 1915-1939. Un épisode de l'histoire de la culture européenne, p. 47-68; «La structure du mot et de l'expression dans l'œuvre de Gustave Chpet, et sa signification pour l'histoire du structuralisme", Slavica occitania, no 26, 2008, p. 13-19. 
entre, d'une part, la démarche philosophique proprement dite, qu'il veut rigoureuse et scientifique, et, d'autre part, certains éléments empruntés à l'orientation religieuse de la pensée russe.

Cette note est elle-même introduite à l'occasion d'une allusion qui est faite dans le texte et qui se rapporte à l'expérience mystique, celle-ci pouvant être considérée comme en dehors de la problématique purement philosophique, mais entretenant, selon Chpet, un lien étroit et particulier avec elle. Il y est question de l'aptitude de tout homme à changer son rapport au monde. En fait, cela doit être mis en rapport avec les réflexions que Chpet vient de conduire sur la structure interne du mot et de l'expression, et sur la possibilité, propre à tout homme, de participer, par cet intermédiaire, à la constitution de la réalité. Ici se cache bien sûr une allusion à la liberté de chaque "Moi», qui ne peut être reconnue en tant que telle qu'à travers les changements que l'individu fait subir au monde qui l'environne, sur la base de ses actes de perception, d'appréhension et d'intellection. Dans un tel contexte, l'intérêt de l'attitude mystique est de permettre la mise en évidence d'un positionnement qui ne nie pas l'acte d'intellection (urazumenie), mais qui le renouvelle fondamentalement.

Chpet écrit à son propos:

En ce qui concerne l'affirmation de l'intellection mystique de l'expérience, sa condition [est] celle d'un problème se rapportant à la satisfaction d'une exigence, avec laquelle l'Esprit appréhendé par la raison entre en action pour se «laisser saisir» ${ }^{15}$.

La référence à l'«Esprit», «Duh» (écrit avec une majuscule et en italique) peut paraitre étonnante dans un texte qui a comme vocation d'introduire, en Russie, un courant de philosophie dite scientifique (la phénoménologie husserlienne). Cependant, un approfondissement de la démarche chpétienne, même si nous nous limitons au seul texte de Le phénomène et le sens, révèle la présence, chez lui, d'une perspective, qui engage l'acte de compréhension dans une visée qui le transcende et l'englobe simultanément. Mais cette perspective, chez Chpet, ne correspond en aucune façon à l'attitude qui est habituellement considérée comme caractéristique de la mystique: une attitude solitaire de retrait par rapport au monde. Pour Chpet, elle témoigne plutôt de cette capacité qu'a l'homme de se détacher de tous les domaines

15. G. Špet, Javlenie i smysl... [Le phénomène et le sens...], p. 211; p. 175: «Мистическое уразумение опыта подразумевает удовлетворение требования, с которым выступает для своего “приятия" сам уразумеваемый Дух». Nous utilisons, en français, la traduction que nous avons faite avec $\mathrm{F}$. Teppe, à paraître prochainement sous le titre: Le phénomène et le sens. La phénoménologie comme science fondamentale et ses problèmes. 
de la culture déjà établis et de s'ouvrir à ce qui en est le fondement, la base proprement constitutive. Cette «exigence», dont il est question dans le texte, correspond au désir d'aller dans les profondeurs de l'être, de s'enraciner dans ce qui maintient tout ensemble, nous pourrions dire encore: dans ce qui garde l'étant rassemblé et permet à chacun de découvrir les fondements du «rationnel» et de se retrouver, par voie de conséquence, dans une relation primordiale de communication avec les autres.

Immédiatement après avoir parlé de l'Esprit, Chpet écrit:

La mise à l'écart de la «solitude» à travers l'unité de la naissance et de la consanguinité de tout le «rationnel» dans l'unité socio-ecclésiale et les motivations de Ses objectivations sont alors en ce sens la colonne de l'Esprit lui-même ${ }^{16}$.

«Ego» («Ses») est écrit avec une majuscule et désigne précisément l'Esprit qui est appréhendé intellectuellement («Urazumevaemyj Duh»), ou, disons plutôt, pour rester dans la perspective développée dans Le phénomène et le sens, et particulièrement dans le chapitre 7, l'Esprit appréhendé selon l'intuition non pas intellectuelle, mais intelligible («intelligibel'naja intuicija»). C'est ici que, pour la première fois, se trouve fait allusion à la sobornost', bien que cela ne soit pas fait à travers l'usage du concept luimême, mais d'une expression y renvoyant («soborno-social'noe edinenie» [«l'unité socio-ecclésiale»]). Dans cette même phrase se trouve utilisé le terme de «stolp» («colonne») - «Stolp Ego [pour «Duha»-M. D.] samogo» («la colonne de l'Esprit lui-même») -, et l'allusion à l'œuvre maîtresse de Pavel Florensky est ici évidente (Stolp i utverždenie istiny ${ }^{17}$, traduit en français par La colonne et le fondement de la vérité $\left.{ }^{18}\right)$. Plus bas, Chpet cite directement Pavel Florensky et s'exprime à son sujet dans la longue note de bas de page dont nous avons déjà parlé ${ }^{19}$.

Pour bien suivre ce que Chpet cherche à dire au sujet de Pavel Florensky, il ne faut pas oublier que, pour Chpet, l'expérience mystique n'est pas une sortie de ce monde; elle est une autre façon de l'appréhender, et elle reste de ce fait en lien étroit avec les fondements mêmes de l'acte d'intellection. Dans sa note, renvoyant à Pavel Florensky, Chpet cite un passage de La colonne et le fondement de la vérité:

16. G. Špet, Javlenie i smysl... [Le phénomène et le sens...], p. 212; p. 175: «Устранение “одиночества" через единство рождения и единокровного всего "разумного" в соборно-социальном единении и мотивации Его объектирований есть в этом смысле и столп Его самого».

17. Dans sa note, Chpet renvoie à l'édition de 1914 (Moscou).

18. P. Florensky, La colonne et le fondement de la vérité, Lausanne, l'Âge d'homme, 1994 (trad. par C. Andronikof).

19. G. Špet, Javlenie i smysl... [Le phénomène et le sens...], p. 212-213; p. 176-177. 
Et des profondeurs de l'âme se lève un besoin irrésistible de prendre appui sur la «Colonne et le fondement de la vérité ${ }^{20}$.

D’une part, il exprime par là son adhésion à certains aspects de la réflexion de Florensky:

Il n'est pas difficile de voir que, selon cette intellection "particulière», il s'agit d'une pensée que nous avons tracée ${ }^{21}$.

Mais, d'autre part, sa critique est claire et tout à fait significative:

Mais pourquoi faut-il satisfaire ce «besoin» et se détourner de là où tout «se brouille» ${ }^{22}$ ?

Chpet reproche précisément à Florensky d'entendre, par « expérience mystique» le fait de se détourner du monde, alors que, pour lui, Chpet, l'expérience mystique (ou l'appréhension mystique) n'est rien d'autre que la forme la plus élevée (nous pourrions dire aussi: la plus profonde) de l'acte d'intellection. Elle est cette façon d'être au monde à travers une intuition particulière, plus puissante que la simple intuition sensible et portant en elle la finalité de l'acte d'intellection. Chpet ne rejette pas l'idée d'un enracinement de l'expérience mystique dans la vie de l'Esprit (de Dieu pour Florensky), mais, pour lui, cela n'est réalisable qu' «après avoir goûté à l'arbre de la connaissance » (du bien et du mal) ${ }^{23}$, c'est-à-dire après avoir désiré connaître le monde et l'avoir connu, et donc, selon le point de vue religieux qui est celui de Pavel Florensky, après avoir succombé au péché originel, après avoir voulu se placer au même niveau que Dieu et avoir transgressé l'interdiction divine.

Les questions que Chpet pose à Pavel Florensky se ramènent finalement à une seule: faut-il se détacher du péché originel ? Le péché est présenté comme "pre-stuplenie» («crime», pour "prestuplenie» qui peut aussi se traduire par "trans-gression»), «dejanie za granicej» (action au-delà de la frontière: "trans-gression de la frontière»), dans le sens qui est sousentendu, nous dit Chpet, quand on parle de «pre-krasnoe» («très beau»), de "pre-mudroe» («très sage»), de «pre-blagoe» («très bon») ${ }^{24}$.

20. Ibid., p. 213; p. 176: «[...] а из глуби души подымается нестерпимая потребность опереть себя на "столп и утверждение истины" .

21. Ibid.: «Не трудно видеть, что в “особом” уразумении, это - проводимая нами мысль ".

22. Ibid.: «Но почему должно удовлетворять эту “потребность” и отвернуться от того, где "все мятется"?».

23. Ibid.: «[...] только "вкусив от древа познания" ".

24. Ibid. Dans tous ces adjectifs substantivés, «très» traduit le préfixe russe «pre». 
Il s'agit de savoir où est la transgression (le crime, le péché) ? Est-elle du côté du monde ou du côté de Dieu? De la part de celui qui connaît le monde ou de la part de celui qui connaît Dieu? Si la situation de l'homme dans le monde se trouve définie comme "pre-stuplenie», alors, l'autre, celle du mystique, «la gratia quae gratis datur» peut ne pas être vue comme «transgression "; mais inversement, si la trans-gression est dans l'excès ("pre-»), dans le dépassement, dans la sortie du monde, alors, peut-être, est-ce la philosophie qui est salvatrice? De quel côté est le Consolateur («Utešitel'»), ou encore l'Esprit Saint (si l'on saisit l'essence de cette présence ["prisutstvija»])? Si la connaissance est l'acte même d'intellection, alors elle réalise et dépasse simultanément le péché. C'est sans doute pour cette raison que Chpet écrit que finalement, pour la philosophie, il n'y a pas de péché25. La chose est claire: ce que Florensky regarde du point de vue de la théologie, Chpet le voit du point de vue de la philosophie. Cela ne signifie pourtant pas que, pour le philosophe, il n'y ait pas d'appréhension mystique. Celle-ci existe, mais pour le philosophe, comme nous l'avons déjà dit, elle est la forme la plus profonde de présence et d'adhésion au monde, nous pourrions dire: l'essence de l'être-là. En cela le philosophe se désolidarise de l'attitude religieuse, selon laquelle nous devons nous détourner de ce monde, dominé par le péché. Pour le philosophe, le péché, s'il existe, est ce "pre-stuplenie», qui se trouve lui-même dans les actes les plus authentiques d'intellection: un acte de transcender qui reste pourtant dans les limites de l'humain. C'est sans doute pour cela que, dans une œuvre comme Le phénomène et le sens, la référence à Pavel Florensky est donnée dans une note, comme si elle renvoyait nécessairement à ce qui se tient à côté, présent dans l'acte, et pourtant en marge du discours philosophique proprement dit. Serait-ce cela le "pre-stuplenie» de la philosophie? Mais alors dans ce "pre-stuplenie» se découvre aussi l' «obščee» («le général»), ou plutôt, comme Chpet le précisera plus tard, dans La conscience et son propriétaire, l'«ob̌̌čnoe» ("ce qui est commun»), c'est-à-dire ce qui réunit, rapproche, rassemble les deux philosophes, c'est-à-dire encore, selon les paroles de Chpet, cette «exigence» ou ce «besoin» («potrebnost'») de l'homme qui aspire à « dépasser ce qui est antinomique, la contradiction qui pénètre et traverse toute chose ${ }^{26}$. La référence à Pavel Florensky est, pour le philosophe, son "pre-stuplenie» («crime», «péché»), c'est-à-dire ce qui, du sein même de la philosophie, le place dans une expérience de la limite, qui peut avoir des modes opposés et même contradictoires:

25. G. Špet, Javlenie i smysl... [Le phénomène et le sens...], p. 212 ; p. 175.

26. Ibid., p. 213; p. 176: В «преодолении антиномичности, противоречия, проникающего вся и все» [dans «le dépassement de l'antinomie essentielle, de la contradiction qui pénètre tout»]. 
Tout d'abord, nous dit-il:

C'est précisément avec le «péché» que tout prend un autre tour. «Le péché est hors-la-loi» dit le Père P. Florensky en citant Jean le Théologien (p. 168), et «le crime est trans-gression de la frontière, du trait, de la limite, de la "loi", "comme l'adultère" [NdT: pour "pre-ljubodejanie», qui comporte le même préfixe que "pre-stuplenie» indiquant la transgression, l'excès] est un acte sexuel au-delà de la limite, en dehors de ce que l'on doit faire ${ }^{27}$.

Mais il ajoute plus loin, dans la même note:

Et voilà encore un «péché» : «Le mari mort rendant visite la nuit à sa veuve éplorée; le fils ou bien la fille revenant secrètement chez ses parents après une longue séparation, le fiancé, apparaissant à sa fiancée, ou bien l'inverse; l'ange de lumière descendant sur l'athlète de la foi, fier d'avoir longuement jeûné; tous ces cas sont de vampirisme et de phénomènes démoniaques...» (p. 698, c'est moi qui souligne)!... Mais, alors, si... mais j'ai peur de le dire $! ?{ }^{28} \ldots$

Et la note se termine par une question, empruntée au mystique religieux qui est pourtant, lui aussi, philosophe:

Mais vers quoi donc conduit l'Esprit, vers la jus-tification [ $k$ o-pravdaniju] ou bien vers la «félicité » et la «mémoire éternelle» (p. 185) ${ }^{29}$ ?

Ainsi, dans Le phénomène et le sens, la référence à Pavel Florensky représente le mode problématique du rapport au religieux, si spécifique de la pensée russe, et qui reste présent jusque dans une démarche qui se veut pourtant purement philosophique: celle de Chpet.

Dans La conscience et son propriétaire, Chpet se tourne beaucoup plus franchement vers la pensée religieuse russe. Les réflexions purement et exclusivement philosophiques, telles qu'elles ont été développées dans Le phénomène et le sens, sont en revanche moins systématiques et plus ancrées dans une réflexion sur des éléments de logique et de psychologie.

27. Ibid.: «Но именно с "Греха" начинается всё по-разному. "Грех есть беззузаконие"берёт о. Флоренский у св. Иоанна Богословв (168), и “Преступление есть пре-ступление за границу, черту, предел 'закона', 'как и пре-любодеяние есть половое деяние за границей, опричь черты должного' (7о0)” ».

28. Ibid.: «А вот тоже “грех": "Умерший муж, посешаюший ночьми свою тоскующую вдову; сын или дочь, таинственно проходящие из далёкой разлуки к родителям; жених, являющийся невесте, или наоборот; ангел света, слетающий к запостившемуся и возгордившемуся подвижнику; - все эти случаи вампиризма и бесо-явления..." (698, подчеркнуто мною)!... Ну, а что, если и... страшно вымолвить!?...».

29. Ibid.: «Но к чему же Дух влечёт, к о-праданию или к “блаженству” и “вечной памяти”? $(185) »$. 
Il est intéressant de voir comment, dans cet écrit de 1916, se trouvent récupérés certains éléments de l'orientation religieuse de la pensée russe, et comment les questions qui ont déjà obtenu une réponse dans le cadre de la philosophie classique proprement dite, sont ici posées d'une façon nouvelle, c'est-à-dire, restent problématiques dans la mesure où elles se trouvent maintenant posées à partir du domaine de la pensée russe.

Dans La conscience et son propriétaire, c'est le problème du «Moi», comme nous l'avons dit, qui se trouve mis en avant. Nous préciserons maintenant: le problème de la conscience personnelle et de la conscience collective. Dans Le phénomène et le sens, ce problème avait été abordé de façon tout à fait secondaire à travers les remarques faites sur l'interprétation de l'expérience mystique, et c'est en tant qu'il restait toujours problématique qu'il pouvait être abordé à partir de réflexions enracinées dans la pensée russe religieuse. Dans La conscience et son propriétaire, nous l'avons déjà évoqué, ce sont les références à Soloviev qui permettent à Chpet de formuler ses propres questions ainsi que son approche critique du subjectivisme. Simultanément, Chpet trouve dans la tradition philosophique religieuse russe confirmation de ses idées sur la priorité à accorder à la conscience collective. Il se tourne vers Serge Nikolaevitch Troubetzkoy qui lui apparait comme le penseur russe le plus approprié pour mettre en valeur, dans la pensée russe, les éléments susceptibles d'être utilisés dans son questionnement philosophique et d'être, par cet intermédiaire, introduits dans l'héritage général de la philosophie. Il écrit à son sujet:

En son temps, dans l'article cité plus haut, S. N. Troubetzkoy avait relevé de nombreux arguments empiriques en faveur du caractère collectif et conciliaire de la conscience. Chez nous, il existe une science qui, quel que soit le nom qu'elle porte, a, comme objet, la conscience ${ }^{30}$.

Et ici commence la citation de S. Troubetzkoy:

[...] [la conscience], dont l'existence ne dépend pas de l'individu. Non point parce que les données de cette conscience présupposent un sujet collectif, différent des individus, constituant un groupe social, mais parce qu'elles ont des caractéristiques que la seule prise en compte des individus en tant que tels ne peut pas permettre d'obtenir ${ }^{31}$.

30. G. Špet, Soznanie i ego sobstvennik [La conscience et son propriétaire], p. 110; p. 307 : «B своё время кн. С.Н. Трубецкой в вышецитированной статье подобрал много эмпирических аргументов в пользу коллективности и соборности сознания. У нас есть наука - как бы она ни называлась - которая имеет своим предметом сознание».

31. Ibid., p. 110-111; p. 307: «[...] [Сознание], существование которого не зависит от индивида. Не потому, что данные этого сознания предполагают коллективный предмет [un sujet collectif], отличный от индивидов, составляющих социальную 
Cette citation est empruntée à l'article de Serge Troubetzkoy, "Sur la nature de la conscience humaine", publié en 1889 dans Questions de philosophie et de psychologie ${ }^{32}$. Nous y trouvons ce que Chpet cherche précisément à mettre en valeur et à définir, mais qu'il ne parvient pas à faire en restant dans le seul cadre de la philosophie classique occidentale. Le concept de «Sobornost' soznanija» (la sobornost' de la conscience) (utilisé en se référant à $\mathrm{S}$. N. Troubetzkoy) renvoie en effet à une démarche particulière, impliquant un rapport au monde spécifique - rapport dans lequel se fondent précisément les réflexions (c'est-à-dire les actes de compréhension et d'intellection) des représentants de la pensée russe religieuse. Pour préciser, Chpet cite Levy-Bruhl (Les fonctions mentales, $2^{\mathrm{e}}$ éd., introduction) qui parle lui-même des «images de l'action, de la pensée et des sentiments, qui présentent cette caractéristique remarquable d'exister en dehors des consciences individuelles ${ }^{33}$.

Cela signifie que, pour le philosophe russe, existe une conscience non individuelle, à laquelle appartiennent certaines idées, ou tout au moins, certains modes de penser ou de sentir, sur la base desquels se détermine l'appartenance des hommes à une modalité de conscience particulière.

Plus loin, Chpet approfondit encore son analyse: le problème du «Moi» et des «Moi» apparaît comme un problème particulier de «l'objet social» qui doit être mis en rapport avec d'autres objets. Dans cet «objet social», il y a une "prédétermination" (prednaznačennost'), mais il y a aussi un principe entéléchique (entelehičeskoe načalo), la «source des vécus et de la conscience, la liberté, la source de ce qui est concret et unique». Et il poursuit: «Ici, la conscience elle-même intervient en tant que ce-donton-a-conscience ${ }^{34}$.

En fait, les éléments qui, dans Le phénomène et le sens, ont permis l'élaboration de la structure du mot et de l'expression, reviennent à la surface. Mais ici, dans La conscience et son propriétaire, ce qui permet le passage de ce-qui-prend-conscience à ce-dont-on-a-conscience (ot soznajuščego do soznavaemogo) est clairement désigné: ce sont les noms propres «imeni sobstvennye». Ce ne sont pas des «imjareki», terme employé ici par Chpet

группу, но потому, что они характеризуются признаками, которых нельзя получить единственно путем рассмотрения индивидов, как таких".

32. S. Troubetzkoï, "O prirode čelovečeskogo soznanija» [ "Sur la nature de la conscience humaine»], Voprosy Filosofii i psihologii [Questions de philosophie et de psychologie], $\mathrm{n}^{\circ}{ }_{1}$, 1889 , p. 91.

33. G. Špet, Soznanie i ego sobstvennik [La conscience et son propriétaire], p. 111; p. 307: «Речь идет об "образах мысли и чувства, которые представляют то замечательное свойство, что они существуют вне индивидуальных сознаний” ".

34. Ibid., p. 113; p. 308 : «Тут само сознание выступает как сознаваемое». 
pour indiquer ceux qui sont désignés par les noms propres mais qui restent toujours en retrait de leur nom, accessibles uniquement par des traces qu'ils laissent dans le monde environnant, au sein d'un domaine d'activités ou de savoir, grâce à leur aptitude à modifier tout donné de la culture. La simple compréhension de ce fait est déjà un indice de participation à cette conscience supérieure et unificatrice qui n'anéantit pas chaque "Moi», mais permet, au contraire, sa réalisation. À la fin de La conscience et son propriétaire, Chpet explique enfin à quel type d'expérience il a voulu faire allusion précédemment, en particulier lorsque, dans Le phénomène et le sens, il renvoyait à Pavel Florensky.

Il écrit: "Ce qui se rapporte à sobornost' représente une sphère autonome de la recherche ${ }^{35}$.

Pour Chpet, ce qui est désigné par sobornost' ne peut être découvert que par l'intermédiaire d'une expérience spécifique, et non point seulement par l'intermédiaire d'un savoir. C'est pour cette raison que le domaine qui est alors investi par ce type d'expérience n'est pas celui des «généralités» ou des «abstractions», mais celui de la "communication " ${ }^{36}$. Ce qui permet d'y accéder, c'est la participation, la communion ${ }^{37}$. L'allusion au sacrement de communion est ici évidente. Elle se place dans la suite de l'interprétation de l'expression de prière «Otče naš» («Notre Père»), qui a été proposée précédemment dans le même texte (p. 113). Nous ne devons pas être étonnés qu'à la fin de ce passage, Gustave Chpet de nouveau se tourne vers la conscience dite religieuse. Il précise:

La conscience religieuse peut être envisagée non seulement comme ayant un caractère général, mais aussi comme appartenant à une communauté ${ }^{8}$.

Pour lui, le domaine du général, c'est le domaine de la forme. Chaque type de conscience (conscience religieuse ou esthétique ou scientifique) a sa forme, qui correspond aux critères de définition et de développement du domaine concerné. Mais ce n'est pas sur cela que veut insister Gustave Chpet. Il est plutôt en quête de ce qui donne sens à tout type de conscience. Il cherche à mettre en valeur cela même qu'il avait appréhendé et présenté dans Le phénomène et le sens comme «acte herméneutique». Dans La conscience

35. G. Špet, Soznanie i ego sobstvennik [La conscience et son propriétaire], p. 114; p. 308 : "Соборное $[. .$.$] есть самостоятельная сфера исследования".$

36. Non pas «obobščenie», mais «obščenie» (ibid., p. 115; p. 309).

37. G. Chpet l'exprime en utilisant les termes de «součastie», «sopričastie», «sopričastnost'» (ibid., p. 114; p. 308).

38. Ibid., p. 115; p. 309: «Сознание религиозное может рассматриваться не только как общее, но и как общное». 
et son propriétaire, c'est un éclairage nouveau qui se trouve donné à la même démarche: la conscience religieuse est nécessaire parce qu'elle se nourrit de l'expérience concrète, qui permet de réunir l'indéterminé et la liberté, c'est-à-dire, pour Gustave Chpet, ce qui est et ce qui se crée. C'est ce type d'expérience, qui, dans Le phénomène et le sens, alors que la conscience n'était pas nommée «religieuse», avait permis de poser la signification, le sens et l'entéléchie comme les trois éléments constitutifs de tout mot, de toute expression, de toute structure sémiotique ${ }^{39}$. Dans La conscience et son propriétaire, au contraire, c'est la référence au religieux qui ouvre la possibilité d'un mode spécifique de la conscience, permettant d'accéder aux formes les plus reculées de l'expérience humaine. Gustave Chpet parle ici de l'activité de l'imrek qui se tient en communauté avec les autres ${ }^{40}$. Déjà, au début de son étude, Gustave Chpet avait ouvert cette voie de compréhension en rejetant la logique aristotélicienne à deux dimensions et en proposant de la remplacer par une autre logique, permettant de sortir du domaine de la pensée rationnelle, dominé par le travail de l'entendement, et de pénétrer dans la profondeur intime de tout être et de toute chose par l'intermédiaire de l'intuition et de la compréhension intelligible de cet intime ${ }^{41}$. Les éléments de cette logique (de cette science du logos), Gustave Chpet les a présentés, dans Le phénomène et le sens - nous l'avons déjà dit -, comme étant l'entéléchie, le sens et la signification.

Dans La conscience et son propriétaire, nous retrouvons une allusion à cela :

La signification et le sens «font obstacle» à la logique, assombrissent sa pureté formelle ${ }^{42}$.

Finalement, pour Gustave Chpet, c'est précisément là que se trouve la clé de tout éclaircissement de l'être, de toute participation à l'être. À un autre moment, il renvoie à la notion d'entéléchie, sous-entendant par «principe entéléchique» ce qui, pour chaque «Moi», pour chaque imrek, est «source

39. À ce sujet, voir en particulier: M. Dennes, Husserl-Heidegger: influence de leur ceuvre en Russie, Paris, L'Harmattan, 1998, et quelques articles du même auteur consacrés à cette question: «La structure du mot et de l'expression dans l'œuvre de Gustave Chpet, et sa signification pour l'histoire du structuralisme»; «De la "structure du mot" à la "forme interne" chez Gustav Špet », Revue germanique internationale [CNRS éditions], n 3, 2006, L'Allemagne des linguistes russes, p. 77-92.

40. G. Špet, Soznanie i ego sobstvennik [La conscience et son propriétaire], p. 101, 103, 106; p. 303, 304, 305 .

41. Ibid., p. 29; р. 268: «Выйти из плоскости рассудочного мышления и проникнуть в глубину, в интимное предмета через уразумения этой интимности ».

42. Ibid., p. 30 ; р. 268 : «Значение и смысл оказываются чем-то “мешаюшим” логике, затемняющим ее формальную чистоту ». 
des vécus et de la conscience ${ }^{43}$. Cela signifie-t-il que l'imrek se définirait totalement par son appartenance à la communauté? Sa participation, sans aucun doute, mais pas son appartenance. À la fin du texte, après avoir une dernière fois renvoyé à Serge Troubetzkoy, afin de confirmer le fait: «En fait concrètement, à propos de tout je tiens en moi-même un concile [sobor] avec tous » ${ }^{4}$, Chpet ajoute: «La ruse ici, ce n'est pas de se tenir en concile avec les autres, mais de se retrouver soi-même à côté de ce concile» ${ }^{45}$.

La question qui surgit est alors la suivante: mais où est-elle, cette liberté de l'imrek («imjarekovaja svoboda»), dont nous parle Chpet? Lui-même insiste sur le fait que la définir ne serait pas raisonnable («nerazumno»). Ce ne serait déjà plus l'imrek. Il écrit: "Sur le terrain de la raison et de l'intellection, une telle "chose personnelle" ne sera déjà plus "mienne", car "le possédé" ce n'est déjà plus le Moi, pas l'imrek, mais la chambre $n^{\circ} n »^{46}$.

Le problème reste irrésolu, et cela est essentiel pour notre sujet:

La tradition de la pensée religieuse russe a permis à Chpet, par l'intermédiaire des héritages de Soloviev, mais aussi de Pavel Florensky, d'Eugène et de Serge Troubetzkoy, de parler d'une façon nouvelle de cette expérience concrète qui se trouve à la base de tout acte d'intellection, et qui, dans un autre contexte, plus essentiellement philosophique, lui avait déjà donné la possibilité de découvrir la structure du mot et de l'expression. Il est clair ici que ces structures sont aussi celles de l'être, mais ce qu'est celui qui, en son acte d'intellection ou de création, les véhicule, reste en dehors de toute appréhension possible. On pourrait parler d'un fond «nominaliste» du réalisme de Chpet, mais c'est en cela, sans aucun doute que s'enracine la liberté fondamentale d'un sujet qui agit pourtant, crée et communique à partir et au sein d'une communauté. Cela correspond-il au fait que, malgré son aspiration à la scientificité, Chpet appartient à une communauté qui a son expérience propre de l'être, et qu'il ne le dénie pas? Nulle part le philosophe ne parle ouvertement de cela, mais l'herméneutique de son œuvre, basée sur une ouverture réaliste qui permet l'existence du fond «nominaliste», dont nous venons de parler, nous autorise à le penser. Et cela est possible dans la mesure où cette appartenance est, pour le philosophe, «nerazumnaja» («irraisonnable»), c'est-à-dire «neizrečennaja» («indicible»); elle est sa «folie», ce qui le possède («oderžimost”»), elle est, rappelons-nous, «pre-stuplenie». Mais en cela se trouvent les conditions de

43. G. Špet, Soznanie i ego sobstvennik [La conscience et son propriétaire], p. 113; p. 308 : «Источник переживаний и сознания ».

44. Ibid., p. 116; p. 310 : « фактически, я по поводу всего держу внутри себя собор со всеми ».

45. Ibid.: «Хитро не "собор со всеми" держать, а себя найти мимо собора».

46. Ibid.: «На почве разума и уразумения, такое “личное” уже и не будет “мое”, ибо “одержимый” уже не Я, не имрек, а палата № $\mathrm{N}$ ”. 
l'existence de l'imrek («imjarekovoe suščestvovanie»). Et nous comprenons alors pourquoi les finales de Le phénomène et le sens et de La conscience et son propriétaire sont toutes deux sibyllines. C'est à l'expérience personnelle, la plus intime et profonde de Chpet qu'elles renvoient, à sa liberté effective et véritable, qu'aucun texte philosophique ne peut exprimer, mais que «justifie» une activité de philosophe qui s'est exercée dans un cadre historique et culturel déterminé.

Maryse Dennes Université Michel de Montaigne - Bordeaux 3 